\title{
Coagulation Factor and Theranostics: A New Paradigm in Molecular Imaging
}

\author{
Jean-Luc C.P. Urbain \\ Lebanon VA Medical Center, Lebanon, Pennsylvania
}

$\mathbf{I}$ n the current issue of The Journal of Nuclear Medicine, Nielsen et al. (1) illustrate the potentials and significant challenges of targeting cancer cells' surface receptors for imaging and therapy.

It is grossly estimated that the human body is made up of about 37 trillion cells (2). These cells communicate with each other through complex networks of extracellular molecules such as growth and inhibitory factors; hormones; neurotransmitters; and cytokines, which bind to specific membrane, cytoplasmic and nuclear receptors. Cell surface receptors are specialized membrane proteins that bind specific cognate ligands and, once activated, relay specific messages to the cell machinery via various molecular cascades under a process called signal transduction.

Although cell surface receptors differ in the way they transmit information into the interior of the cell, in mammalian cells, most receptors can be generalized into 3 distinct and large families based on the mechanisms they use to transmit their signals: ion channel-linked receptors, which convert chemical signals to electrical ones; G-protein-linked receptors that trigger internal

\section{See page 89}

switches; and enzyme-linked receptors that directly activate biochemical activity. In turn, the initiated intracellular signaling cascades regulate numerous cellular functions such as cell division, cell growth and development, apoptosis, and metabolism. The defects or alterations in these proteins at the transcription, translation, or posttranslational levels usually result in disease.

The type and amount of receptors at the surface of normal healthy cells essentially depends on the functions of the cells. Comprehensive profiling studies of the surface proteomes have shown that the membranes of cancer cells undergo significant molecular changes associated with malignization. An increase or decrease in the number of surface receptors, structural changes of membrane receptors that no longer react with their cognate ligand, changes in enzymatic activity, the presence of new surface molecules characteristic of the embryonic state, and abnormal surface molecules that can act as antigens can be observed in cancer cells (3).

Received Sep. 14, 2015; revision accepted Sep. 14, 2015.

For correspondence or reprints contact: Jean-Luc C.P. Urbain, Lebanon VA Medical Center, 1700 S. Lincoln Ave., Lebanon, PA 17042.

Published online Sep. 24, 2015.

COPYRIGHT (c) 2016 by the Society of Nuclear Medicine and Molecular Imaging, Inc.

DOI: $10.2967 /$ jnumed.115.165852
In the past 3 decades, receptor-mediated tumor targeting has received major attention in the field of cancer drug delivery. Comprehensive profiling of the cell surface proteome provides an effective approach for the identification of commonly occurring proteins as well as proteins with restricted expression patterns in cancer (4). Monoclonal antibodies, monoclonal antibody fragments, and drug-loaded delivery systems conjugated with targeting moieties - that is, ligand - are now being used successfully for the targeting of tumors (5). Although having its own specific challenges related to their labeling with isotopes and detection, tumor receptor targeting for imaging and radionuclide therapy largely reflects the theranostics principle approach.

Whereas the field of tumor receptor imaging is best illustrated by the success story of the somatostatin-somatostatin receptors ligand for the diagnosis and treatment of neuroendocrine tumors, hundreds of radiolabeled ligands targeting specific receptors have been tested in animals and in clinical trials. In this innovative research endeavor, Nielson et al. describe the use of the tissue factor (TF) transmembrane glycoprotein as a potential imaging receptor target and surrogate marker for the assessment of its own inhibition with chemotherapeutic agents.

$\mathrm{TF}$, also known as factor III, thromboplastin, or CD142, is a 47-kDA membrane-bound glycoprotein encoded by the F3 gene on chromosome 1 that is expressed on subendothelial cells and that is essential for vascular hemostasis. It binds the coagulation serine protease factor VII/VIIa and forms a bimolecular complex that serves as the primary initiator of coagulation in vivo in the extrinsic coagulation pathway. TF is expressed at high levels by the adventitial fibroblasts of the vessel walls to facilitate a rapid coagulation cascade. It is also expressed in a variety of highly vascularized tissues such as the brain, heart, kidney, and placenta. Low expression is seen in the spleen, thymus, and liver. Serum TF is derived from its expression in blood cells, microparticles containing TF shed from cells, and soluble TF protein. TF appears to play a role in wound repairs, embryonic development, cell adhesion, angiogenesis, and innate immunity. TF can be induced in smooth muscle cells and endothelial cells of the vessels in various disease conditions such as sepsis, diabetes, cardiovascular disease, and cancer, resulting in a prothrombotic state. The close link between coagulation and diseases also suggests a complex interplay between the clotting cascade and disease progression (6).

The prothrombotic effect of TF was first described in the 19th century by Armand Trousseau, who mentioned thrombophlebitis as a complication of pancreatic cancer. The risk of thrombosis and thrombophlebitis is about 4 times higher in patients with cancer than in the general population. The incidence of thrombosis is also greater in patients with tumors expressing a higher level of TF 
such as brain and pancreatic cancers. Increased circulating levels of TF, protocoagulant plasma membranes (MPs) shed from cancer cells, the induction of TF expression in monocytes and endothelial cells that also release MPs, and the increased expression of TF by chemoradiation treatments are suspected to have a major role in the hypercoagulable state of cancer patients (7).

TF is expressed by virtually all types of cancers. It has been implicated in cancer-associated thrombophlebitis, metastasis, tumor growth, and tumor angiogenesis. TF expression in cancer cells is the result of well-defined upstream oncogenic transformation events. In colorectal cancer, for example, the constitutive activation of the MAPK and PI3K signaling pathways triggered by the mutation of the K-ras oncogene and p53 tumor suppressor gene leads to the enhanced expression of TF. The amplification of the epidermal growth factor receptor and the tumor suppressor gene PTEN also modulates the expression of TF at the surface of cancer cells. The protooncogene Src tyrosine kinase and the transforming growth factor $\beta$ also stimulate the induction of TF expression (8).

Tumor angiogenesis and migration seem to be favored by TF (9). Studies have reported that increased TF levels in tumor cells is associated with increased expression and levels of the vascular endothelial growth factor and fibroblast growth factor 2. Tumor progression is also facilitated by TF, which activates thrombin generation, fibrin deposition, platelet activation, tumor-associated macrophage recruitment, and metastasis. After activation by FVIIa, TF promotes tumor cell proliferation via the protease-activated receptor 2 signaling cascade and through integrin signaling. Cytoskeleton remodeling and modulation of the immune response within the tumor microenvironment are also attributed to TF.

These numerous roles underscore the interest of TF in therapy and imaging. Antitumor TF strategies have been developed in a few directions. One involves chimeric proteins consisting of targeting and procoagulant domains, which recognize the tumor vasculature and the thrombogenic site and induce thrombosis within the tumor vasculature and the necrosis of the tumor. A second approach used the TF receptor as the main target and the FVII conjugated with the Fc effector domain of human IG1 to kill cells expressing TF with direct injection into the tumor to maximize the chemotherapeutic effect and minimize bleeding (6). More recently, small-interference RNAs have been used to silence the expression of TF in lung and colorectal tumors (10).

The labeling of the TF receptor with FVII represents another example of the vibrant molecular imaging research that is based on the characterization of the membrane proteome and the targeting of cancer cell surface receptors. It also illustrates nicely the complexity and integrative aspects of normal and complex pathophysiologic cellular and molecular biology mechanisms.

The detection and imaging of TF is a relatively new concept. It has been used for the detection of acute bleeding with labeling of the activated FVII with ${ }^{99 \mathrm{~m}} \mathrm{Tc}$ and ${ }^{111} \mathrm{In}(11,12)$, the ex vivo imaging of unstable atheromatous lesions that are rich in macrophages (13), and more recently, the imaging of TF overexpression in a subcutaneous xenograft mouse model using an anti-TF antibody labeled with ${ }^{99 \mathrm{~m}} \mathrm{TC}$ and ${ }^{64} \mathrm{Cu}$, respectively (14).

In the article in The Journal of Nuclear Medicine, Nielsen et al. used the active site-inhibited blood-clotting factor VIIa (FVIIai) to image in vitro and in vivo cancer cells and tumors expressing the TF transmembrane receptor. Factor VIIa is a vitamin K-dependent
50-kDa trypsinlike serine protease that plays a pivotal role in hemostasis and coagulation by binding to TF in a bimolecular complex. It circulates in plasma in zymogen form and becomes activated on binding to the TF. The inactivation of the active site of FVII by the incorporation of a small peptide inhibitor results in the blockade of the cleavage of FVII into a 2-chain serine protease that activates the extrinsic coagulation pathway while preserving and actually enhancing its binding to TF.

Using state-of-the-art radiolabeling, molecular biology techniques, a subcutaneous xenograft mouse model, and an animal hybrid imaging scanner, the authors demonstrated an elegant and promising approach for the labeling, visualization, and quantification of $\mathrm{TF}$ expression at the surface of cancers cells with ${ }^{18} \mathrm{~F}$ FVIIai.

Although this method warrants in vitro and in vivo confirmation and further development, the potential of moving into PET clinical trials with ${ }^{18} \mathrm{~F}$-FVIIai to detect, pinpoint, and quantify a common but potentially useful molecular tumor receptor (similarly to the successful ${ }^{18}$ F-FDG PET glucose transporter story) while assessing the efficacy of novel chemotherapeutic agents is quite interesting and appealing.

\section{DISCLOSURE}

No potential conflict of interest relevant to this article was reported.

\section{REFERENCES}

1. Nielsen CH, Erlandsson M, Jeppesen T, et al. Quantitative PET imaging of tissue factor expression using ${ }^{18} \mathrm{~F}$-labeled active site-inhibited factor VII. J Nucl Med. 2016;57:89-96.

2. Bianconi E, Piovesan A, Facchin F, et al. An estimation of the number of cells in the human body. Ann Hum Biol. 2013;40:463-471.

3. Baba Al, Câtoi C. Comparative oncology. In: Tumor Cell Morphology. Bucharest, Romania: The Publishing House of the Romanian Academy; 2007.

4. Leth-Larsen R, Lund R, Ditzel H. Plasma membrane proteomics and its application in clinical cancer biomarker discovery. Mol Cell Proteomics. 2010;9: 1369-1382.

5. Mankoff DA, Link JM, Linden HM, et al. Tumor receptor imaging. J Nucl Med. 2008;49(suppl 2):149S-163S

6. Kasthuri RS, Taubman MB, Mackman N. Role of tissue factor in cancer. J Clin Oncol. 2009;27:4834-4838.

7. van den Berg YM, Osanto S, Reitsma P, et al. The relationship between tissue factor and cancer progression: insights from bench and bedside. Blood. 2012;119: 924-932.

8. Chu AJ. Tissue factor, blood coagulation and beyond: an overview. Int J Inflam. 2011;2011:367284

9. Han X, Guo B, Li Y, Zhu B. Tissue factor in tumor microenvironment: a systematic review. J Hematol Oncol. 2014;7:54.

10. Xu C, Gui Q. Chen W wt al. Small interference RNA targeting tissue factor inhibits human lung adenocarcinoma growth in vitro and in vivo. $J$ Exp Clin Cancer Res. 2011;30:63.

11. Madsen J, Kristensen JB, Olsen $\mathrm{OH}$, et al. Recombinant coagulation factor VIIa labelled with the fac- ${ }^{99 \mathrm{~m}} \mathrm{Tc}(\mathrm{CO})_{3}$-core: synthesis and in vitro evaluation of a putative new radiopharmaceutical for imaging in acute bleeding lesion. $J$ Label Compd Radiopharm. 2011;54:214-219.

12. Nalla A, Buch I, Sigvardt M, Bodholdt RP, Kjaer A, Hesse B. ${ }^{111}$ Indium labelling of recombinant activated coagulation factor VII: in vitro and preliminary in vivo studies in healthy rats. Int J Mol Imaging. 2012;2012:464810.

13. Temma T, Ogawa Y, Kuge Y, et al. Tissue factor detection for selectively discriminating unstable plaques in an atherosclerotic rabbit model. $J$ Nucl Med. 2010;51:1979-1986.

14. Hong H, Zhang Y, Nayak TR, et al. Immuno-PET of tissue factor in pancreatic cancer. J Nucl Med. 2012;53:1748-1754. 